\author{
MIKOŁAJ NKOLLO \\ Université Adam Mickiewicz de Poznań \\ mink@inea.pl
}

\title{
LES ASPECTS ÉVOLUTIFS DES EXPOSANTS DE LA RÉCIPROCITÉ MÉDIÉVAUX. ANCIENNES ET NOUVELLES MÉTHODES DE RECHERCHE
}

\begin{abstract}
Mikołaj Nkollo, Les aspects évolutifs des exposants de la réciprocité médiévaux. Anciennes et nouvelles méthodes de recherche [Evolutionary paths of Old French reciprocal markers. New and old methodological tools], Studia Romanica Posnaniensia, Adam Mickiewicz University Press, Poznań, vol. XXXIX/4: 2012, pp. 99-112. ISBN 978-83-232-2510-2. ISSN 0137-2475. eISSN 2084-4158.
\end{abstract}

The present paper revolves around unusual paths of grammaticalization of Old French $\left(12^{\text {th }}\right.$ century) reciprocal markers. This methodological framework requires medieval means of expressing reciprocity to be compared both with their parent forms in Classical Latin and with the markers introduced in subsequent stages of the history of French language. The first hypothesis deals with how parallel markers, i.e. ones that have a common origin and that are used inside the same area of grammar (se... entre- and entre eux $<$ Lat. inter se), are different from each other. This path is claimed to materialize provided one of the two terms begins to serve a particular function not performed by the other one. The second hypothesis accounts for what means were used to prevent ongoing reflexive / reciprocal homonymy. This task happened to be provisionally fulfilled by cors a cors and coste a coste until the advent of adverbs ending in -ment in $14^{\text {th }}$ century. As a consequence, body-part nouns lost most of their grammatical potential. The third hypothesis, formulated in terms of exaptation, explains how and why languages are likely to recycle erstwhile peripheral lexical elements. This evolutionary path takes place in response to a need to convey a given meaning unambiguously. Attempts at avoiding reflexive / reciprocal homonymy prompted the revival of seemingly forgotten Latin items reciprocus / mutuus. Concluding remarks address the problem of whether the concept of exaptation is useful in historical linguistics and contain a proposal towards constraining its scope. As for current views of grammaticalization, this notion seems to call for further refinements, as well.

Key words: grammaticalization, exaptation, recycling, evolution, reciprocity, reflexive $v s$ reciprocal

\section{BUT ET MÉTHODES}

Le présent article concerne les méthodes de recherche sur l'évolution des langues naturelles. Le renouvellement de l'intérêt pour cet aspect des études linguistiques est dû surtout au succès de la notion de grammaticalisation. Formulée par A. Meillet 
(1921 : 133), rappelée et raffinée par J. Kuryłowicz (1965 : 68), elle explique à l'aide de ses paramètres l'origine de pratiquement tous les moyens auxiliaires utilisés dans les langues humaines. Seront rangés, parmi ces moyens, les signes, indépendamment de leur caractère peu ou fortement grammatical, qui contribuent à l'expression des concepts fonctionnels. Définis par opposition aux concepts lexicaux, les concepts fonctionnels englobent $p$. ex. le mode verbal, les marqueurs de relations discursives (cause, but, concession, hypothèse, etc.), la flexion casuelle, la gradation des adverbes, etc. L'origine des moyens qui transmettent toutes ces significations, même de ceux qui ont aujourd'hui un caractère affixal, remonte, dans la théorie de grammaticalisation, à des unités qui représentaient anciennement des mots à part entière et qui avaient au départ un caractère lexical. Le cas emblématique d'une telle évolution est illustré par les changements qui ont affecté l'ablatif du nom latin mens, mentis. Précédé d'un adjectif, ce terme, dont les caractéristiques initiales étaient par excellence celles d'un mot autonome, a fini par "dégénérer» au rang de partie d'un autre mot. Sa signification ancienne a glissé vers une signification purement fonctionnelle (celle d'un suffixe adverbialisateur), ce qui s'accompagnait de la perte de ses anciens privilèges morphologiques et syntaxiques (la faculté d'être fléchi, de fonctionner comme tête d'un syntagme, etc.).

Le but de cet article est d'éclairer certaines questions litigieuses liées justement à la théorie de grammaticalisation. Le débat sera articulé autour des auxiliaires de réciprocité découverts à l'issue de la lecture des textes français médiévaux. L'observation des exemples, recueillis dans deux poèmes épiques et une chanson de geste rédigés au XII ${ }^{e}$ siècle - Perceval ou le conte du Graal (CGr), le Roman de Thèbes (RdT) et le Couronnement de Louis (CL) - permettra de vérifier le bien-fondé des hypothèses formulées tant au sujet du parcours même du processus, qu'à propos de l'origine des marqueurs de la réciprocité.

En premier lieu, les réflexions porteront sur un cas de coexistence à l'intérieur de ce segment de la grammaire de deux exposants de réciprocité descendus d'une même source. Le fonctionnement en parallèle des signes à étymologie commune qui concordent à transmettre un même sens échappe généralement aux spécialistes. L'analyse menée dans les pages qui suivent se rapportera aussi à l'émergence des moyens capables d'écarter l'homonymie des verbes pronominaux mis en rapport syntaxique avec un SN coordonné ou pluriel. Graduellement réalisée, cette évolution ne s'est achevée qu'à l'aube du français moyen. L'étude des solutions adoptées entre-temps offre également l'occasion à un examen attentif de certaines constatations émanant des autorités de la théorie de grammaticalisation.

Enfin, pour répondre aux questions devant lesquelles cette théorie reste impuissante, un autre concept sera évoqué, celui d'exaptation. Apparu d'abord dans la biologie évolutive ${ }^{1}$, il a été adroitement introduit dans les recherches linguistiques. À partir

\footnotetext{
${ }^{1}$ Sa définition, dont la paternité est attribuée à deux biologistes, Gould et Vrba, est la suivante : «We wish to restrict the term adaptation only to those structures that evolved for their current util-
} 
des années quatre-vingt-dix, il ne cesse pas de se frayer le chemin en diachronie et s'accomode parfaitement de l'étude des mécanismes évolutifs dans les langues humaines. Comparée à la grammaticalisation, l'exaptation commence toujours après coup, c.-à.-d. au moment où un élément lexical ancien avait déjà subi les changements paramétriques - érosion phonétique, occurrences régulières dans des contextes nouveaux, désémantisation, altération de ses propriétés morphosyntaxiques. Il peut donc s'agir de l'acquisition des fonctions grammaticales nouvelles par une unité qui avait déjà fini de se grammaticaliser. Alternativement, si l'évolution n'a pas abouti à la mise en place d'un ensemble efficient de moyens auxiliaires, agissent d'autres mécanismes susceptibles de parer aux besoins communicatifs des usagers. Il peut se produire, dans ces circonstances, des glissements sémantiques autres que ceux auxquels s'intéresse la théorie de grammaticalisation. Sont aussi fréquents des emprunts avec des distorsions de sens inattendues. Ce dernier scénario, nommé parfois « recyclage », est attesté dans l'évolution des marqueurs de réciprocité médiévaux.

\section{LA RÉCIPROCITÉ MÉDIÉVALE FRANÇAISE - VARIANTES STRUCTURALES}

La réciprocité a un double mode d'existence dans les langues humaines. En premier lieu, cette signification est propre à des expressions que la tradition logique appelle « noms de relations symétriques », usage souvent repris par les linguistes. Il est question ici des unités lexicales qui, indépendamment de leur partage en parties du discours, renvoient à des états de choses réciproques. Y appartiennent entre autres : mariage, pourparlers, contraste, contigu, analogue, se chamailler, parier, copiner, etc. Par la suite, ces noms seront considérés comme formant une classe lexicale sémantiquement homogène et morphologiquement hétérogène. Peu de choses seront dites à leur propos. L'autre méthode de signaler la réciprocité consiste à attacher des éléments auxiliaires appropriés à des expressions dont le sens n'a au départ rien à voir

ity; those useful structures that arose for other reasons, or for no conventional reasons at all, and were fortuitously available for other changes, we call exaptations » (citation d'après Traugott, 2004 : 133). Les cas emblématiques de l'évolution survenue sur la base d'un organe qui s'est initialement développé pour un usage différent comprennent: 1) les poumons des tétrapodes, formés à partir des branchies de certains organismes aquatiques vivant dans des milieux pauvres en oxygène, qui ont été secondairement modifiés pour fonctionner dans un milieu terrestre ; 2) les pattes des tétrapodes, qui ont remplacé les nageoires, ont été développés avant la sortie des eaux et la conquête de la terre; peu à peu, elles ont commencé à servir à agripper les proies lors d'une attaque. Il semble que la fonction de déplacement réalisée au moyen de pattes, qui a permis de quitter les eaux et de conquérir la terre ferme, a apparu plus tard ; 3 ) le plumage, qui servait au début à une meilleure régulation thermique durant la course, au camouflage et dans les parades sexuelles, a subi une exaptation graduelle pour le vol ; 4) le lait des mammifères qui est probablement produit par des glandes sudoripares modifiées. Une sécrétion proche de la sueur devait suinter sur le corps des ancêtres des mammifères ; à leur naissance, les jeunes venaient se blottir à leur mère et étaient exposés au contact avec cette sécrétion aux vertus nutritives. 
avec ce concept. Ces éléments, à caractère soit lexical (adverbes, adjectifs), soit grammatical (clitiques, anaphoriques bipartis, affixes, etc.), figurent obligatoirement dans des combinaisons réciproques - respect mutuel, s'admirer l'un l'autre, amour entre eux, interdépendance, etc. Est ainsi réalisée la conjonction d'au moins deux relations non symétriques de même nature, inversement orientées. C'est cette deuxième classe de signes qui constitue l'objet propre des réflexions qui suivent. Les versions intégrales des trois textes médiévaux français offrent sept types de combinaisons réciproques, illustrées et caractérisées ci-dessous :

1. Les constructions où la conversion s'appuie sur le pronom se. Joint à peu près toujours à une forme verbale plurielle, cet élément était responsable d'une double interprétation, réfléchie ou réciproque, de la phrase. Comparé à son continuateur contemporain, le pronom médiéval disposait d'une mobilité plus grande. Dans des séquences verbales comprenant la forme personnelle d'un verbe auxiliaire (temporel, modal, aspectuel) et la forme non finie du verbe sujet à la conversion, il s'attachait alternativement à l'une ou à l'autre d'entre elles.

Lor morz se pardonent et plorent RdT 5759

Les compaignes s'apruisment tant

Que les guardes qui vont davant

Se comenciérent a mesler RdT 7569-7571

Au departir se corurent besier CL 241

Mout se penerent de rades cops ferir CL 2520

2. Les constructions appuyées sur un auxiliaire discontinu composé de se et du entre- préfixé au verbe ${ }^{2}$. Il arrive que différentes versions d'une même œuvre offrent tantôt des fragments où la conversion est réalisée par l'emploi de se seul, tantôt des phrases où le même effet est obtenu par la combinaison de se avec le préfixe en question. En témoigne le vers 241, évoqué ci-dessus, du Couronnement de Louis, qui dans un des manuscrits préservés figure sous la forme Au departir se sont entrebesiez. Curieusement, le Conte du Graal contient deux occurrences des formes verbales préfixées par entre-, mais non accompagnées de se. Puisqu'il s'agit des formes imper-

${ }^{2}$ Suivant les affirmations de Z. Guentchéva et de N. Rivière (2007 : 605-607), la mobilité du préfixe entre- dans les textes anciens lui permettait de s'attacher à un verbe autre que celui qui était sujet à la conversion. Dans des séquences composées de la forme finie d'un verbe auxiliaire et d'une forme non-personnelle de l'unité lexicale transformée, l'emplacement de entre- oscillait entre l'une et l'autre forme. Les deux linguistes appuient leur opinion sur les exemples suivants (le numéro du vers n'est disponible dans aucune de leurs citations) : Il s'entrecommencent a regarder (Chrétien de Troyes, XII ${ }^{\mathrm{e}} \mathrm{s}$ ), Le peuple s'entrevoullaient tuer (Renart XIII' ${ }^{\mathrm{e}} \mathrm{s}$.) ou Quant il s'entrecuidaient ferir (Couronnement de Louis, XII ${ }^{\mathrm{e}} \mathrm{s}$.). La version de cette dernière œuvre, compilée à partir des quatre manuscrits actuellement connus, dont proviennent les fragments évoqués dans le présent article et qui est accessible sur le site de l'Université d'Ottawa, ne contient pas la phrase citée par Guentchéva et Rivière. Voir < http://www.uottawa.ca/academic/arts/lfa/activites/textes/Couronnement/coltexte. $\mathrm{htm}>$. 
sonnelles coordonnées entre elles dans une subordonnée, cet exemple doit être regardé comme exceptionnel. La combinaison se... entre-, qui semble représenter une innovation française, répandue ensuite à d'autres langues romanes, résulte de la réanalyse du groupe prépositif latin inter se. Son emploi dans les poèmes médiévaux est une parfaite illustration de grammaticalisation. La préposition inter, ancien élément libre combinable avec les formes de l'accusatif, a peu à peu acquis le statut de partie d'un autre mot.

Puis s'entresgardent et se moutrent le vis CL 2518

Uns vavasors andemantiers

antra leanz, qui mout lor nut,

qui monseignor Gauvain conut,

si les trova antrebeisant

et mout grant joie antrefeisant. CGr 5759-5763

3. Les constructions où le rôle auxiliaire échoue à entre eux, l'autre continuateur, non grammaticalisé, de inter se. Contre l'usage moderne, la préposition était parfois sujette au changement, sans altérer le caractère réciproque de la totalité. À l'opposé de la combinaison précédente, cet exposant est bien attesté dans d'autres langues romanes de la même période. Cependant, se... entre- et entre eux ne doivent pas être mis sur un pied d'égalité - certaines différences discursives les séparent. Puisque leur fonctionnement jette une lumière nouvelle sur l'évolution des auxiliaires de réciprocité anciens, ils seront caractérisés dans la partie consacrée à la grammaticalisation.

que par l'amor et par la foi

qui doit estre antre li et moi. CGr 5630-5631

Et tant com pueent por eus orent $\mathrm{RdT} 5760$

Cil dui plaidierent entr'els mout longement CL 2444

4. Les combinaisons où la conversion est réalisée grâce à l'emploi de l'expression bipartite chascuns l'autre. Peu attestées dans les textes anciens, les phrases ainsi construites oscillaient entre une interprétation réciproque et une autre, que l'on pourrait caractériser comme séquentielle. Cette dernière, du fait de faire appel à l'idée d'ordre linéaire, représente dans le meilleur des cas une variante modérée de réciprocité (Dalrymple et al., 1998 : 168-169). En raison de son paradigme défectif (avec le pluriel non attesté), chacun l'autre figure, dans tous les exemples enregistrés, en compagnie des formes du singulier verbal. Chacun des deux éléments de cet exposant représente une autre forme casuelle. Il n'est donc pas étonnant de découvrir qu'ils apparaissent à l'intérieur des deux nœuds syntaxiques distincts d'une phrase.

Li uns plaint l'autre, grant duel font;

Chascuns plaint l'autre plus que sei RdT 5754-5755

5. Les constructions avec l'anaphorique biparti li uns... l'autre (cas sujet - cas régime). Cette combinaison, beaucoup plus attestée que la précédente, pose cependant encore plus de problèmes interprétatifs. Il n'est pas rare de rencontrer les phrases 
où elle est attachée à un verbe non accompagné de se. Cette dernière variante a lieu chaque fois que la forme verbale figure au singulier. La lecture réciproque n'est définitivement retenue que là où le verbe soumis à la conversion ne fait pas référence à l'idée d'ordre linéaire (matérialisée, p.ex., par des paires d'unités converses : supérieur - inférieur, précéder - suivre), ceci à condition que soient engagés exactement deux participants. Ce cas de figure est illustré par les deux premiers exemples.

Iluec se voleient combatre

Li uns contre l'autre et abatre. RdT 10169-10170

\section{Li.i. a l'autre le voloient noncier CL 198}

Tuit le virent par le tornei,

L'uns a l'autre le mostre al dei RdT 5463-5464

Se commenciérent a mesler,

Li un les autres a ruser $\mathrm{RdT} 7571-7572$

6. Les constructions où l'expression de la réciprocité repose sur les locutions formées d'après le modèle : nom d'une partie du corps - préposition - nom d'une partie $\mathrm{du}$ corps. Tous les exemples s'appuient sur la réitération des mêmes noms (seuls cors et coste sont attestés dans les œuvres évoquées ici) mis en correspondance par la préposition $a$. Cette méthode d'expression de la réciprocité est préservée actuellement. Cependant, sa position dans les écrits médiévaux n'était pas identique à celle des locutions contemporaines telles que coude à coude, nez à nez, tête à tête, etc. Au Moyen Âge, ces exposants étaient chargés de combler la lacune due à l'absence, jusqu'au $\mathrm{XIV}^{\mathrm{e}}$ siècle, des adverbes réciproques terminés en -ment. C'est ainsi qu'était neutralisée l'homonymie : réfléchi - réciproque occasionnée par le pronom se. Ce mode d'expression de la réciprocité s'observe dans plusieurs langues humaines (Heine \& Miyashita, 2008: 182-185), y compris les différents stades du latin³. La répétition d'une partie de la phrase ou la réduplication de l'un de ses termes représentent iconiquement le caractère complexe des situations signifiées par les combinaisons réciproques.

${ }^{3}$ L'expression de la réciprocité en latin reposait entre autres sur la répétition des différentes formes d'un nom, localisées l'une directement à côté de l'autre (le nominatif, toujours obligatoire, suivi par l'autre forme casuelle, déterminée chaque fois par les propriétés combinatoires de l'expression convertie). Puisque dans tous les exemples recueillis jusqu'à présent ces noms figurent en emploi générique, leur référence n'est que virtuelle. Ils nomment tout au plus les participants potentiels à une situation. En témoignent des constructions comme Placet Stoicis homines hominum causa esse generatos... ou Cives civibus prodesse opportet. En outre, le caractère conjonctif des états de choses mutuels était signalé par des syntagmes bâtis sur deux formes flexionnelles juxtaposées d'un même pronom indéfini. Étaient ainsi formés des groupes comme : uterque utrumque (latin préclassique, p.ex. Uterque utriquest cordi), alius alium, alter alterum, les deux derniers bien attestés à la période classique (Alios alii deinceps excipiebant ou Fratres alter alterum adiutant). Dans les commentaires normatifs sur la grammaire latine (Gildersleeve et Lodge, 1895/2003 : 154), l'opposition entre les deux derniers exposants est caractérisée de la façon suivante : alter alterum décrit ce qui se passe à l'intérieur d'un groupe composé de deux individus et alius alium s'applique à des collectivités plus nombreuses. 
En effet, il s'y agit toujours de la conjonction d'au moins deux instances, différemment orientées, des relations d'un même type appliquées aux mêmes individus. Le mécanisme conceptuel découvert derrière ces locutions est clair : à une duplicité des parties (« sous-événements » de Nedjalkov, 2007 : 151-152) des états de choses réciproques correspond une double occurrence des noms de leurs participants ou des noms d'objets anaphoriquement associés à ces participants.

Messire Gauvains coste a coste

fist devant lui mangier son oste CGr 7962-7963

En l'ost de Troie fu defors,

Et se combatié cors a cors

O Eneas, qui fu mout proz,

Fors Hector, li mieudres de toz RdT 7235-7238

7. Les constructions polyphrastiques qui transmettent ouvertement l'information sur le caractère biparti des situations en question. De ce fait, elles sont indépendantes de l'existence dans une langue des auxiliaires de réciprocité. Puisque le français médiéval ne disposait pas d'adverbes chargés de reprendre la première partie de la construction dans la direction inverse, les locuteurs devaient chercher d'autres moyens pour éviter les redites. La solution est venue du côté des pronoms. Anaphoriquement introduits dans un texte, ils suffisaient à identifier les participants à la situation. Sujets à moins de contraintes que leurs continuateurs contemporains, ils avaient la faculté, qui les sépare très manifestement des pronoms actuels, de ne pas être directement attachés à la forme verbale. Leur soumission à l'hôte était réduite au point que devenaient acceptables les groupements des clitiques représentant des formes casuelles distinctes.

Qant messire Gauvains l'esgarde,

d'aler contre li ne se tarde,

si la salue et ele lui. CGr 7859-7861

Vos confondez lui et il vos RdT 7917

\section{CONSÉQUENCES POUR LA THÉORIE DE GRAMMATICALISATION}

Le premier cas jugé pertinent pour la discussion sur la théorie de grammaticalisation concerne le fonctionnement en parallèle et à l'intérieur d'un même segment de la grammaire du continuateur direct du terme-parent et de la forme grammaticalisée qui en descend. Bien attesté dans les poèmes écrits au Moyen Âge, ce scénario met en cause le bien-fondé des définitions qui associent la grammaticalisation à la déperdition du terme source. Les auxiliaires rattachables à un même étymon comprennent le groupe entre eux et la forme discontinue se... entre- (préfixe). L'un et l'autre signe remontent à inter se, syntagme utilisé en latin pour exprimer la réciprocité (Aristides et Themistocles obtrectarunt inter se ;... valde enim inter se amabant propter inorum 
similitudinem). Ont été mariées grâce à l'existence simultanée de ces auxiliaires deux tendances apparemment contradictoires - le besoin d'innovation et celui de conservation. La question se pose alors de savoir s'il s'agit d'un encombrement, d'un couple pléonastique à un membre inutile et amputable au besoin ou d'un dédoublement prémédité à vocation précise.

La réponse à cette question sera plus facile, si les fonctions discursives anciennes des auxiliaires en question sont analysées. Jointes aux unités lexicales à sens non-réciproque, les expressions se... entre- et entre eux partageaient au Moyen Âge l'aptitude à faire ressortir le caractère mutuel d'une situation. Là où il s'est agi de transformer un nom, la phrase contenait de préférence un support verbal (existentiel, causatif ou autre).

Venge le vers ceus qui mort l'ont

Et qui entre eus grant joie en font $\mathrm{RdT} 6869-6870$

Trist et marri, car tal rien mut

Par quei entre eus grant ire crut RdT 7855-7856

Mout par se sont entremaumis,

Or se sont andui entrocis RdT 9661-9662

Il s'entraloignent dous arpenz $\mathrm{RdT} 9617$

Il s'entrebesent neveu sont et ami CL 1479

Cependant, dans d'autres contextes, les fonctions prises en charge par entre eux se rapportaient davantage à l'opposition : distributif $v s$ collectif. En effet, une phrase comme Ils rivalisent d'arrache-pied pour gagner la course, où les noms des participants appartiennent à un même nœud (sont coordonnés ou réduits à un élément pluriel), signifie soit que l'un des individus identifiés par le pronom rivalise avec l'autre individu nommé par cet élément (la phrase est complète), soit qu'il y a rivalité entre le référent collectif de $i l s$ et quelqu'un d'autre, qui n'est pas ouvertement nommé (la phrase est incomplète). Par contre, Ils rivalisent entre eux d'arrache pied pour gagner la course a uniquement la première interprétation. Cette valeur sémantique est bien illustrée dans les écrits médiévaux.

\section{Entre eus se vont ja discordant}

Et de lor regne contendant, $\operatorname{RdT} 520-521$

Entre eus en meinent mout grant plait RdT 327

En revanche, la sélection de l'interprétation où les mêmes rôles sont distribués entre tous les participants à la situation identifiés par le syntagme nominal n'est qu'exceptionnellement assurée par se... entre-. En termes du nombre des occurrences, cet effet peut donc passer pour propre à entre eux, plutôt qu'à se... entre-. En effet, les textes évoqués ici n'offrent qu'un exemple où ce dernier auxiliaire est attaché au nom d'une relation symétrique. 
La coexistence des auxiliaires à étymologie commune et leur fonctionnement en parallèle à l'intérieur d'un segment de la grammaire représentent deux aspects de l'évolution passés sous silence par la théorie de grammaticalisation. Premièrement, entre eux et se... entre- prouvent que l'émergence d'un auxiliaire à caractère grammatical n'équivaut pas toujours à la mort du terme source. Celui-ci peut, en effet, avoir son continuateur direct qui fait son service à côté de l'unité nouvelle. Les deux éléments contribuent dans ce cas-là à transmettre des significations fort proches. Deuxièmement, ce scénario se réalise uniquement à condition de la spécialisation de l'une des expressions (Dahl, 2001 : 114-116). Employé pour informer que la distribution des rôles se fait à l'intérieur de l'ensemble identifié par le syntagme nominal, entre eux assume ainsi une fonction hautement spécifique que son homologue est pratiquement incapable de remplir. Cette opposition a permis, à ce qu'il semble, de maintenir l'un et l'autre exposant au cours des siècles.

L'autre observation au sujet du développement des exposants de la réciprocité concerne l'homonymie : réfléchi - réciproque. Il s'agit, plus précisément, des moyens dont disposait le français médiéval afin de l'écarter. Les chercheurs qui reconstruisent l'évolution des termes auxiliaires dans les langues humaines, associent l'origine des exposants de la réciprocité à l'une des trois sources : a) auxiliaires de réflexivité ; b) noms évoquant le concept de corps; c) noms à sens « ami », « compagnon». Les deux premiers mécanismes sont bien représentés dans les textes médiévaux.

En ce qui concerne la première voie évolutive, les linguistes notent l'imminente confusion des deux interprétations, réfléchie et réciproque, à l'intérieur des phrases comme Ils se pardonnent tout ou Les automobilistes s'injuriaient et s'apostrophaient. Une des explications les plus convaincantes de cette homonymie est issue de la plume de D. Creisselss (2006, ch. 22) qui l'interprète en termes de la duplicité des rôles assumés par les participants à certaines situations. Plus spécifiquement, il s'agit, selon lui, de l'extension de cette duplicité à partir des singletons à des ensembles plus nombreux. Tant que cette duplicité concerne un élément, la situation a un caractère réfléchi - son unique initiateur représente, en même temps, son unique point d'aboutissement. La confusion surgit dès que la duplicité en question intéresse au moins deux individus ; chacun est capable d'initier la situation soit envers lui-même, soit envers l'autre. Ce deuxième scénario donne naissance à des interprétations réciproques. Au niveau formel, cette proximité conceptuelle est matérialisée par la tendance à employer le même auxiliaire pour l'un et l'autre effet sémantique. Certains éléments spécialisés écartent, en français contemporain, la lecture non désirée. Le tri est effectué, pour signaler le sens réfléchi, par l'adjonction de même à la forme tonique du pronom personnel et par l'emploi de réciproquement / mutuellement, pour insister sur la réciprocité.

Les deux derniers termes ne sont pas attestés dans les écrits médiévaux français. La sélection de l'interprétation récipoque était, en leur lieu et place, assurée par les constructions bâties d'après le modèle : nom d'une partie du corps - préposition nom d'une partie du corps. Les locutions cors a cors, coste a coste, qui comblaient 
la lacune due à l'absence des adverbes capables d'écarter le réfléchi au profit du réciproque, illustrent donc le deuxième mécanisme évolutif postulé par la théorie de grammaticalisation. Ce scénario semble d'ailleurs strictement tributaire de la réflexivité. Puisque le concept de son corps représente un substitut commode du concept de " soi-même », il constitue une source précieuse d'exposants de la réflexivité (Heine \& Miyashita, 2008 : 174-176). Celle-ci sert, à son tour, selon le mécanisme analogique dépeint par Creissels, à signaler le caractère réciproque d'un état de choses. Bien que cette source soit identifiée comme réutilisation des noms signifiant " corps » en guise d'auxiliaires de réciprocité (Heine \& Kuteva, 2002:58 et les chercheurs qu'ils évoquent excluent les noms de ses parties), les méronymes relatifs au corps humain se rangent également dans cette catégorie.

Cette voie évolutive représente une instance de grammaticalisation arrêtée à michemin. Un cycle grammatical reste inachevé sans un changement préalable de la signification de l'unité lexicale ancienne. Ce changement, appelé « désémantisation » (semantic bleaching) s'accomplit lorsqu'un élément « se vide progressivement de son sens plein pour acquérir en revanche un sens fonctionnel, grammatical » (Lamiroy, 1999 : 35). Les noms de parties du corps n'ont jamais gagné le statut d'un exposant universellement applicable de la réciprocité. Leur valeur sémantique initiale a été gardée à titre de signification principale au cours de toute l'histoire. Le sens réciproque n'est intervenu qu'à titre d'un effet secondaire. Les emplois auxiliaires des noms de parties du corps ne sont attestés qu'auprès des verbes de mouvement ou de ceux qui renvoient à un combat. La référence y était donc faite à des activités physiques, exécutées avec les parties du corps. Par contre, les combinaisons comme être jaloux nez à nez ou se haïr dos à dos ne s'observent ni dans les textes médiévaux ni contemporains. Le pas décisif n'a donc pas été fait vers l'autre paramètre de grammaticalisation, appelé « élargissement », qui consiste dans l'emploi d'une unité ancienne dans une série de contextes nouveaux (cf. Heine \& Song, 2011 : 591). Avec l'introduction dans le lexique des adverbes terminés en -ment, les noms de parties du corps ont été relégués aux périphéries du système d'expression de la réciprocité.

\section{EXAPTATION ET RECYCLAGE - NOUVEAUX OUTILS D'ANALYSE EN DIACHRONIE}

Au Moyen Âge la répartition des rôles entre des auxiliaires particuliers de réciprocité avait un caractère fluctuant. Les méthodes anciennes étaient donc, pas à pas, abandonnées au profit d'un système plus stable. Pourtant, certains aspects de cette évolution échappent à l'analyse conduite en termes de grammaticalisation. Restent peu claires surtout les causes de l'épanouissement ultérieur, en guise de termes capables de sélectionner l'interprétation réciproque, des unités nouvelles inconnues au XII ${ }^{\mathrm{e}}$ siècle. La question se pose de savoir si les adverbes réciproquement et mutuellement 
représentent une innovation abrupte, apparue ex nihilo ou bien s'il y a eu une chiquenaude qui a initié leur émergence. En dépit de leur incontestable ancrage empirique, la théorie de grammaticalisation et ses paramètres n'ont pas cette fois-ci grand-chose à dire à propos de leur entrée dans le lexique français. L'explication de la progression vers l'état moderne exige donc que soit cherchée une solution alternative.

Les noms coste et cors, peu ou nullement désémantisés, n'insistaient pas avec suffisamment de force sur l'interprétation réciproque de certaines séquences. Joints uniquement aux noms d'activités physiques, ils n'en sont jamais venus, pas plus que d'autres noms de parties du corps, à exprimer la réciprocité auprès des verbes à référents abstraits. La dualité réfléchi - réciproque continuait donc son règne. Quant à l'un l'autre, introduit dans des contextes syntagmatiques très hétérogènes et difficilement applicable pour convertir les noms ou les adjectifs, il ne suffisait parfois pas à transmettre commodément la réciprocité. Sa lourdeur se manifestait en outre dans des constructions réciproques à valeur possessive. La pression pour se procurer une méthode efficace d'écarter d'éventuelles homonymies a donc précipité la quête des moyens nouveaux.

La solution est venue du côté des termes empruntés au latin. Ont été ainsi ressuscités des termes longuement oubliés - les adjectifs reciprocus et mutuus. Transformés en adverbes, ils ont pris, à partir du XIVe siècle, la relève des noms de parties du corps jugés peu efficaces pour sélectionner les interprétations réciproques. L'autre catalyseur de l'émergence de ces unités, consécutif aux changements survenus dans la prosodie, peut être associé à la nécessité d'asseoir sur des critères nouveaux l'organisation des constructions polyphrastiques. À partir du XII ${ }^{e}$ siècle, l'ancien accent mobile, pouvant affecter différentes syllabes des mots particuliers localisés à pratiquement tous les endroits de la phrase, était graduellement éliminé au profit d'un accent immobile (Marchello-Nizia, 1995 : 187-189 ; Zaring, 2010 : 13-14). Assigné à chaque groupe rythmique plutôt qu'aux mots qui le formaient, cet accent nouveau a désormais interdit d'appuyer la partie localisée derrière la conjonction sur les pronoms en emploi libre, c.-à.-d. non attachés directement à une forme verbale (cf. Vos confondez lui et il vos, RdT 7917). Cependant, la volonté d'éviter les redites qui se produisent lorsqu'on nomme deux relations inversement orientées, mais concernant les mêmes individus (Marie aime Jacques et Jacques aime Marie) a favorisé l'emploi des unités nouvelles, jusque lors inconnues, chargées d'assurer le même effet. Puisqu'ils permettaient d'éviter les réitérations des segments voisins d'un texte, les adverbes recyclés ${ }^{4}$, réciproquement et mutuellement, se prêtaient parfaitement à cette fin.

${ }^{4}$ Parmi les tentatives d'appliquer la notion d'exaptation à la description des langues naturelles, une des meilleures réussites est due à D. Weiss (2005). Dans son étude des participes adverbiaux polonais (formes terminées en -qc et -wszy), il a démontré les changements en train de se poursuivre dans leurs emplois. Peu usitées avec leur valeur canonique (coréférence de leur « sujet sous-enten$\mathrm{du} »$ avec celui de la principale, contraintes sur la localisation temporelle des situations auxquelles les deux propositions renvoient), ces formes sont pourtant souvent recyclées. Certains participes de cette classe sont de plus en plus attestés à l'intérieur des locutions adverbiales, prépositives et conjonctives. La propriété commune à la presque totalité de ces usages nouveaux concerne le 
C'est pour décrire un changement où la fonction actuelle d'un organe n'est pas celle qui lui échouait primitivement (avant que ne se fasse sentir la pression de l'évolution) que le terme " exaptation » a été forgé. Ainsi rend-t-il compte des situations où un dispositif biologique ancien est réutilisé, après une série de « bricolages », pour une fonction non sélectionnée à l'origine. Initialement apparue dans la biologie, cette notion a été rapidement introduite en linguistique (Heine et Kuteva, $2007: 8-9$ ).

En ce qui concerne la mise en place de réciproquement, il s'agit d'un transfert réalisé, à ce qu'il semble, à dessein. Empruntée au latin classique, la base adjectivale $\mathrm{y}$ fonctionnait avec une valeur sémantique à peine liée à la réciprocité, à savoir « qui revient au point de départ 》 (cf. reciprocae voces - « échos », reciprocum mare « mer qui reflue »; Gaffiot, 1934). Son emploi en guise d'auxiliaire représente donc une instance de recyclage - reciprocus a été réutilisé après des siècles avec un sens complètement nouveau et dans des contextes anciennement non attestés (il est surtout vain de le chercher dans les séquences polyphrastiques latines).

Moins de détours sémantiques (mais autant d'innovations syntaxiques) accompagnaient l'introduction dans le lexique national de l'autre terme délibérément sorti de l'oubli, l'adjectif mutuus. Absent dans des textes médiévaux les plus anciens et refait du coup en adverbe grâce à la suffixation populaire en -ment, cet élément hybride renferme un radical très bien attesté en latin classique. Fréquemment ajouté à inter $s e$, il figurait surtout dans des constructions réciproques possessives (cf. Olores mиtua carne vescuntur inter se - lit. « dévorent entre eux leurs chairs mutuelles »; ... pro mutuo inter nos animo - lit. «à cause de notre affection de l'un pour l'autre »). Était ainsi faite concurrence à unus alterum, également employé dans ce type de fonctions (Alter alterius judicium reprehendit). Apparu dans d'autres contextes (cf. Inter se mortales mutua vivunt), mutuus continuait à fonctionner à côté de inter se. En français il a radicalement changé de son comportement syntaxique et de ses caractéristiques fonctionnelles. Une fois ressuscité, il s'employait à partir du XIVe siècle surtout : a) dans des constructions polyphrastiques, $b$ ) dans des séquences avec un verbe pronominal pour écarter la réflexivité (se détestent mutuellement) et c) en compagnie des adjectifs

caractère métatextuel des séquences discursives où ces participes figurent. Il s'agit des interventions délibérées du locuteur dans l'organisation de son propre texte, de la volonté de se justifier, des appels faits à la mémoire discursive des auditeurs, etc. Weiss a identifié une série d'écarts par rapport à la norme que présentent ces emplois nouveaux. Il s'agit de l'effacement des oppositions aspectuelles (poczynajac od ...., a kończqc na vs poczqwszy od... a skończywszy na où aucune différence sémantique ne sépare les formes perfectives et imperfectives), de l'érosion du rôle de la négation (nie wspominajac o...), de la perte de la coréference entre les entités évoquées (Zważywszy na jej sytuację, radziła sobie całkiem fajnie au lieu de Zważywszy na swoja sytuację, radziła sobie całkiem fajnie où le changement du possessif témoigne de la non coréférence). Weiss ne se sert pas dans son article du terme " exaptation ». Par contre, il emploie volontiers celui de " recyclage » défini comme : fonctions nouvelles qui assurent la survie de certaines unités représentant une catégorie globalement vouée à l'extinction. 
- noms de relation soumis à la transformation réciproque (des raisonnements mutuellement exclusifs). Il ne manifeste plus la même faculté qu'il avait en latin d'apparaître en compagnie de entre eux.

\section{CONCLUSION}

Nouvellement apparu en linguistique, le concept d'exaptation devra encore faire ses preuves dans les recherches à venir. Pour l'instant, son attrait consiste à profiler le fonctionnement d'une langue naturelle à l'image de celui d'un organisme vivant. Il a aussi le mérite de montrer que dans leur évolution les composantes de la grammaire ont plutôt tendance à bricoler qu'à suivre un plan préétabli. Rien donc d'étonnant que plusieurs chercheurs ne résistent pas à la volonté de se servir de cette notion. L' « exaptation » recouvre actuellement, au gré des options théoriques des auteurs, des phénomènes assez hétérogènes. Un bon exemple en est l'idée selon laquelle elle représenterait l'étape terminale d'une instance de grammaticalisation (cf. Traugott, 2004 : 143, « Exaptation in language change can be thought of as the phenomenon of the emergence of a new grammatical function at what could otherwise be expected to be the end of a cline of grammaticalization »). Il y a parmi ces phénomènes ceux qui peuvent être décrits avec succès au moyen de l'appareil notionnel offert par la théorie de grammaticalisation. Les emplois abusifs de la notion d'exaptation font stagner les recherches diachroniques plus qu'ils ne contribuent à leur progrès. Il est donc désirable de restreindre son emploi uniquement à ces changements qui échappent aux paramètres traditionnels. Une tentative dans cette direction a été entreprise dans cet article.

Forgée il y a à peu près un siècle, la notion de grammaticalisation ne cesse pas de résister à l'épreuve du temps. Ont été expliquées grâce à elle l'origine et la grande diversité des auxiliaires, y compris ceux dont l'émergence a suivi des scénarios très particuliers, qui ne se répètent pas d'une langue à l'autre. Pourtant, les recherches historiques fournissent toujours des exemples de changements survenus de manière inattendue et difficilement prévisible. Deux cas de ce type ont été décrits ici : un cycle grammatical arrêté à mi-chemin (noms de parties du corps) et l'existence en parallèle des formes rattachables à une même source dont l'une a subi la grammaticalisation et l'autre non. Les développements futurs de la théorie de grammaticalisation devront tenir compte de ces instances d'évolution qui s'écartent du schème général.

\section{BIBLIOGRAPHIE}

Creissels, Denis (2006): Syntaxe générale. Une introduction typologique. 2 : La Phrase. Paris : Hermès science-Lavoisier.

DAHL, Östen (2001): « Grammaticalization and the life cycles of constructions ». RASK - Internationalt tidsskrift for sprog og kommunikation 14 : 91-134. 
Dalrymple Mary, Kanazawa, Makoto; Kim ,Yookyung; Mchombo, Sam; Peters, Stanley (1998): « Reciprocal Expressions and the Concept of Reciprocity ». Linguistics and Philosophy 21 (2): 159-210.

Gaffiot, Félix (1934): Dictionnaire latin-français. Paris : Hachette.

Gildersleeve, Basil Lanneau; Lodge, Gonzalez (1895/2003): Gildersleeve's Latin Grammar. Wauconda : Bolchazy-Carducci Publisher.

Guentchéva, Zlatka; Rivière, Nicole (2007): « Reciprocal and reflexive constructions in French ». In : Vladimir P. Nedjalkov et al. [ed.]. Reciprocal Constructions, vol. 2. Amsterdam - Philadelphia: John Benjamins Publishing Company, 561-607.

Heine, Bernd; Kuteva, Tania (2002): World Lexicon of Grammaticalization. Cambridge : Cambridge University Press.

Heine, Bernd; Kuteva, Tania (2007): The genesis of grammar: a reconstruction. Oxford : Oxford University Press.

Heine, Bernd; Miyashita, Hiroyuki (2008): «The intersection between reflexives and reciprocals: A grammaticalization perspective ». In : Volker GAST, Ekkehard KöNIG [eds.]. Reciprocal and Reflexives, Theoretical and typological explorations. Berlin - New York : Walter de Gruyter, 169-223.

HeInE, Bernd; Song, Kyung-An (2011): « On the grammaticalization of personal pronouns ». Journal of Linguistics 47 : 587-630.

KurYŁowicz, Jerzy (1965): «L'évolution des catégories grammaticales ». Diogène. Revue internationale des sciences humaines $51: 54-71$.

LAmiroy, Béatrice (1999): «Auxiliaires, langues romanes et grammaticalisation ». Langages 135 : $33-45$.

Marchello-Nizia, Christiane (1995): L'évolution du français : Ordre des mots, démonstratifs, accent tonique. Paris : Armand Colin.

MeILlEt, Antoine (1921): Linguistique historique et linguistique générale. Paris : H. Champion.

Nedjalkov, Vladimir P. (2007): « Encoding of the reciprocal meaning ». In: Vladimir P. Nedjalkov et al. [ed.]. Reciprocal Constructions, vol. 1. Amsterdam - Philadelphia : John Benjamins Publishing Company, 147-207.

Traugott, Elizabeth C. (2004): « Exaptation and Grammaticalization ». In : Minoji Акiмото [ed.]. Linguistic Studies Based on Corpora. Tokyo : Hituzi Syobo Publishing Co., 133-156.

WeIss, Daniel (2005): « Nowe przyimki o pochodzeniu imiesłowowym? ». In : Maciej GrochowsKI [ed.]. Przystówki i przyimki. Studia ze składni i semantyki języka polskiego. Toruń : Wydawnictwo Uniwersytetu im. Mikołaja Kopernika, 177-207.

ZARING, Laurie (2010): " Changing from OV to VO: More evidence from Old French ». Ianua. Revista Philologica Romanica 10 : 1-18. 